\title{
Employing the patient-centered collaborative care approach: a case study of complex geriatric patient with psychopathalogy of treatment resistant depression and primary hyperparathyroidism.
}

\author{
Joel R Saul ${ }^{1}$, Sharon M Holder ${ }^{2,3 *}$, Jonathan S Lokey ${ }^{3}$ \\ ${ }^{1}$ Department of Psychiatry and Behavioral Science, Greenville Health System, South Carolina, USA \\ ${ }^{2}$ Department of Public Health Sciences and the Institute of Family and Neighborhood Life, Clemson University, South \\ Carolina, USA
}

${ }^{3}$ Department of Psychiatry \& Behavioral Medicine, Greenville Health System, South Carolina, USA

\begin{abstract}
Introduction: Primary hyperparathyroidism is a relatively common endocrine disease with many nonspecific physical symptoms as well as potentially serious psychiatric effects. The incidence of the illness, severity of symptoms, and poorer outcomes are all positively correlated with increasing patient age. The Patient Centered Collaborative Care Approach is a demonstrable method of coordinating patient care, reducing the burden on patient advocates, and improving patient outcomes. The use of the Patient Centered Collaborative Care Approach has not previously been well described in treatment of psychiatric manifestations of primary hyperparathyroidism.

Methods: Using the example of an elderly woman admitted with complex psychopathology and a diagnosis of primary hyperparathyroidism, this case will illustrate the value of the Patient-Centered Collaborative Care Approach in (1) establishing accurate diagnoses, (2) reducing harm from comorbidities, (3) improving patient outcomes, (4) potentially reducing overall hospital costs.

Results: The case exemplifies the potential benefits of implementing the Patient-Centered Collaborative Care Approach in the treatment of a medically complex patient hospitalized for psychiatric complaints.

Conclusion: A diagnosis of Primary hyperparathyroidism (PHPT) was not immediately made due to variables confounding initial lab values to include significant dehydration and malnourishment prior to admission. A multidisciplinary team was employed in the treatment for this patient utilizing the Patient centered Collaborative Care Approach. This included the psychiatric treatment team, internal medicine, endocrine surgery, as well as significant family involvement. Parathyroid adenoma was confirmed mass was successfully removed surgically. The patient made a rapid and dramatic improvement and her depressive symptoms remitted. The critical importance of clinical flexibility, questioning available data, and a collaborative team approach to the evolution of a diagnosis is discussed.
\end{abstract}

Keywords: Geriatric psychiatry, Hypercalcemia, Parathyroidectomy, Patient-centered collaborative care, Primary hyperparathyroidism (PHPT), Treatment resistant depression.

Accepted on 02 January 2018

\section{Introduction}

Primary hyperparathyroidism (PHPT) is a disorder of calcium metabolism resulting in abnormally high levels of serum calcium secondary to increased levels of parathyroid hormone $[1,2]$. Primary hyperparathyroidism is also considered one of the most prevalent endocrine diseases with an incidence rate of approximately 3 per 1,000 in the general population and up to 1 per 100 in the elderly; with older females being the most frequently affected [3]. Though the typical presentation of PHPT involves presence of renal stones, bone loss, and gastrointestinal complaints, screening of serum calcium during routine medical examination has expanded the clinical spectrum to include patients with minimal objective symptoms who are referred to as asymptomatic [4]. Psychiatric manifestations associated with hyperparathyroidism include

depression, anxiety, and disorders of thought and behavior; with depression often presenting as persistent sadness, apathy, decrease in self-care, change in appetite, and anhedonia [5].

Primary hyperparathyroidism is easily misdiagnosed if not clinically suspected and appropriate biochemical tests carried out. The differential diagnosis for hypercalcemia is broad and includes, in addition to PHPT: malignancy, hypervitaminosis D, immobility, drugs such as lithium and thiazide diuretics, chronic renal failure, and hyperthyroidism to name a few. It is critical to obtain PTH levels in any patient with hypercalcemia [2].

The risk of developing PHPT increases with age, and older patients experiencing PHPT are likely to display a range of neuropsychiatric symptoms including treatment resistant 
depression (TRD), psychosis and reduced neurocognitive functions [5]. Recent studies have confirmed biochemical markers of PHPT to be characterized by a number of other symptoms formerly uncharacteristic of the condition $[6,7]$. Such symptoms include low energy, fatigue, poor sleep, weakness [8] bone and joint pain, poor nutrition and vitamin deficiencies.

The consensus is the natural history of PHPT proposes that the symptoms of hyperparathyroidism progress in approximately $40 \%$ of patients, particularly as one aged [9]. Hence, as the life expectancy in older people increases, the more likely there will be a diagnosis of PHPT. This becomes especially important for management of the disease because those living with PHPT may be at higher risk for the development of complication in comparison to younger cohorts. This places geriatric patients at increased risk for comorbidity and poor outcomes. However, studies show improvement in subjective neuropsychiatric symptoms post PHPT, although most have utilized institutional survey-based tools to assess pre-and postoperative symptoms [10].

Using the example of a real case presentation of a 79 year old woman, (referred to as Mrs. Brown, a pseudonym) admitted with complex psychopathology and a diagnosis of PHPT, this case presentation will illustrate how the impact of collaboration between the patient, family and healthcare professionals can enhance the quality of patient care by utilizing the patientcentered collaborative care model, including the lessons learned.

\section{Patient Centered Collaborative Care}

It has been recognized that patient-centered collaborative care is a desired and necessary part of health care delivery and an important element of quality patient care $[11,12]$. For example, the Institute of Medicine (IOM) strongly recommended patient-centered care rather than disease-centered or clinician driven care. Patient-centered is one of the six tenets for improvement in the IOM 2001 report, crossing the Quality Chasm, emphazing that patient-centered care responds to the individual patient's preferences, needs and values, meriting that patient input is incorporated in clinical decisions (IOM 2001). Building on this tenet, patient-centered collaborative care is made possible through a conscious service alignment tailored to meet the needs of the patient population served by at least two healthcare providers working collaboratively with patients and their caregivers, to the extent preferred by each to accomplish shared goals within and across settings to achieve coordinated, high-quality care [13]. Conversely, for the patientcentered collaborative care model to be understood fully, family members and decision makers must become active partners in multi-disciplinary decision making and care. These individuals often have first-hand insight into patients' preferences and can make important contributions to care decisions, particularly when patients are unable to advocate for themselves. Patient-centered collaborative care is increasingly perceived as "the moral thing to do," and is considered by many to be justified on ethical grounds $[14,15]$.
However, this model is not without criticisms. Though a number of studies have demonstrated that patient-centered collaborative care is associated with better clinical outcomes, improved physician-patient communication and relationships $[11,12,14]$, higher patient satisfaction, better recall of information and treatment adherence, better recovery as well as satisfaction of patients and providers [16,17], the model is not without critics. Some argue instead for 'rational continuity', that is, the on-going, caring relationships between the patient and their known provider continuing in the future and linking their past care with current care without a cadre of people involved $[18,19]$. Others have argued that the patient-centered collaborative care model may focus too much on increasing staff and capacities to build partnerships [20-26], yet lacking continuity and increasing focus on infrastructure and processes such as health information technology. It has also been argued that the patient-centered collaborative care model must go beyond the health systems because people with mental health issues and other co-morbidities have needs that go beyond health, transcending across the life-course [21].

\section{Background and Patient-Centered Collaborative Care Approach}

The Greenville Health System (GHS) is a not-for profit nationally recognized academic health center committed to advancing patient care through research, scholarship, education and clinical excellence. The Department of Psychiatry and Behavioral Medicine is an integral part of GHS. The Department provides services for a broad range of psychiatric disorders and includes services at the Marshall I. Pickens Hospital (MIPH) and the GHS Outpatient Psychiatry clinic. Patients with psychiatric disorders are usually treated based on collaboration between healthcare providers with the primary aims of addressing the needs of individual patients and improving patient outcomes. Patient-centered collaborative care in the department involves participation among healthcare providers including input from psychiatrist, social workers, physicians, pharmacists, nurses, hospital chaplain, nutritionist and psychiatry residents aimed at improving outcomes in meeting the needs of mentally ill patients.

The program takes a collaborative care team approach to treating our psychiatric patients. That is, an approach that facilitates inter and intra sharing of knowledge between the patient, family members, healthcare professionals, specialists and collaborators in meeting the needs and care of our patients. Treatment includes immersion in a total therapeutic environment including but not limited to the following: individualized treatment and care plan, individual and group therapy sessions, activity and recreational therapy sessions, and individual educational program in response to individual patient's and family preferences, needs and values.

\section{Case Presentation}

Mrs. Brown (a pseudonym, Mrs. Brown and patient will be used interchangeable throughout the case presentation) is a 79 year old, married, retired, white female who was admitted to our psychiatric hospital for suicidal ideation in the setting of 
Citation: Saul JR, Holder SM, Lokey JS. Employing the patient-centered collaborative care approach: a case study of complex geriatric patient with psychopathalogy of treatment resistant depression and primary hyperparathyroidism. J Ment Health Aging 2018;2(1): $18-23$.

severe, treatment resistant depression, anxiety, nihilistic thinking, and significant guilt that had a delusional quality. Mrs. Brown was admitted following an exacerbation of depressive and anxious symptoms for which she was seen twice by an outpatient psychiatrist prior to admission. Mrs. Brown stated that her symptoms began approximately one year ago after moving with her husband from another state, and coupled with the unexpected development of her husband's increasing health issues. She stated that she became her husband's primary caregiver and she quickly became overwhelmed with the amount of work involved. Prior to the development of her symptoms she had been very active and healthy for her years and was a competitive tennis player until shortly prior to her move. The patient was a retired veteran and also worked as a physical therapist for years as well as a homemaker. Prior to moving she was very active in her community and frequently visited friends. After moving, her family noted she began to isolate herself and ceased all social activity.

However, this was the first psychiatric admission for Mrs. Brown and her past psychiatric history was minimal; including 3-4 remote panic attacks. She denied any history of psychosis, mania, significant depression or anxiety. The patient had been in good physical health and had a medical history of wellcontrolled hypertension and hypothyroidism, as well as a history of cardiac arrhythmia, cataracts and occasional incontinence. On admission, patient appeared very depressed and spoke in a low whisper with some notable hoarseness. Patient endorsed a history of dysphagia to liquids and solids. A diagnosis of PHPT was not immediately made due to variables confounding initial lab values to include significant dehydration and malnourishment secondary to patient refusing by mouth intake for several days prior to admission in an attempt to starve her to death.

Mrs. Brown's chief complaint at admission was: "I'm depressed, have been for a long time." She endorsed feeling chronically overwhelmed, depressed and anxious, significantly decreased sleep and appetite, anergia, anhedonia, feelings of overwhelming guilt that at times seemed to have a persecutory delusional quality, and passive suicidal ideation. She endorsed severe feelings of hopelessness and helplessness and frequently ruminated on thoughts that she was "beyond salvation" for reasons she described as having "doomed my family, I have ruined us all." However in discussion with her family members this was not in fact true. Mrs. Brown stated that as depression worsened she began having more cognitive trouble particularly with memory and her ability to complete day-to-day tasks. Neurocognitive disorders such as Alzheimer's dementia were not foremost on the initial differential due to the rapid onset of patient's mood symptoms with cognitive issues only developing once her depression was quite severe; a diagnosis of PHPT was identified prior to a dementia workup or neuroimaging being necessary. On admission patient complained of vague lower abdominal and pelvic discomfort and was found to have a urinary tract infection as well as acute kidney injury secondary to dehydration.

\section{Admitting medication}

The patient was admitted on Lisinopril $20 \mathrm{mg}$ tablet by mouth daily in morning, Sertraline $100 \mathrm{mg}$ tablet by mouth daily in morning, Clonazepam $1 \mathrm{mg}$ tablet by mouth daily at bedtime, Cyanocobalamin $500 \mu \mathrm{g}$ tablet by mouth daily and morning, Cholecalciferol 1000 unit tablet by mouth daily in morning, L. Acidophilus 10 billion cell capsule by mouth daily in morning and Multivitamin tablet by mouth daily in morning.

\section{Patient-centered collaborative treatment}

Mrs. Brown was admitted to the inpatient geriatric psychiatry unit that was adequately staffed for the observation and treatment of geriatric patients with psychiatric illness. The facility has the capability to manage some medical concerns on site such as IV fluids, and multiple medical and surgical subspecialties are available for collaboration on a consultant basis. The patient's presenting complaint of depression and anxiety was complicated by multiple physical symptoms and metabolic derangements as well as the patient's refusal to eat or drink. The dual, initial treatment goals were to optimize psychiatric medication to treat symptoms while simultaneously treating her medical illnesses. In this facility physical examinations are completed by a medical physician or physician extender and the collaborative treatment team includes social work, recreational therapy, nursing, hospital chaplain, nutritionist and a psychiatric attending/resident team. Treatment team meetings were held regularly during which all aspects of individual patient care are discussed as a group and optimized. Emphasis is placed on family involvement and collateral history gathering when available. The patient initially isolated to her room, lying in bed for most of the day in the dark, though she was eventually willing to spend more time in the dayroom though minimally involved in the milieu. During the course of Mrs. Brown's hospitalization we maintained frequent communication with members of her involved family via telephone and collaborative meetings, keeping them abreast of developments and progress. The patient was daily engaged and encouraged by members of the collaborative team; seeking her thoughts and opinions and incorporating her preferences where feasible.

\section{Pharmacologic, Behavioral and Surgical Interventions}

After admission the patient was tapered off of Sertraline 100 $\mathrm{mg}$ and started on Mirtazapine $15 \mathrm{mg}$ by mouth at bedtime to encourage both appetite and sleep; as well as to eliminate the potential for gastrointestinal side effects from Sertraline further confusing the clinical presentation. Due to the patient's severe anxiety, she was weaned off of Clonazepam $1 \mathrm{mg}$ in favor of 1 $\mathrm{mg}$ of Lorazepam at bedtime and $0.5 \mathrm{mg} 3$ times a day. Also, because of the severity of patient's depression prior to admission and her symptoms presentation on the unit, the team recommended electroconvulsive therapy (ECT) and the consult was placed. As illustrated in previous studies [22,23] the ECT was deemed appropriate and patient went through 4 sessions while further workup was being completed. ECT treatments were performed with bi-temporal electrode placement, 
achieving adequate seizure during each session. Mrs. Brown continued to refuse food and hydration and due to limited insight and impaired judgment a temporary inability to consent was completed for her with family involvement. Mrs. Brown's kidney function normalized after intravenous (IV) replacement of fluids and her urinary tract infection was treated with oral antibiotics by the medical nurse practitioner on staff. Her parathyroid intact hormone levels had been within the highnormal range and calcium was elevated (Table 1); however, after IV fluid resuscitation, calcium levels remained elevated and a medical consult was placed for more detailed workup. Ultrasound of patient's neck was performed which revealed a 9 $\mathrm{mm}$ well-defined hypoechoic soft tissue nodule present along the deep surface of the inferior pole of the right thyroid. This correlated with a nuclear medicine sestamibi scan of the parathyroid ordered by the collaborating internist. It was suspected that the patient had a hyperfunctioning parathyroid adenoma; ECT was subsequently discontinued and endocrine surgery was consulted.

Table 1. Laboratory examination at admission and preparathyroidectomy.

\begin{tabular}{lll}
\hline Lab Value & At Admission & At Admission \\
\hline Calcium (Ref: 8.5-10.4 mg/dl) & 13.5 & 10.3 \\
\hline Calcium lonized (Ref: $4.5-5.3 \mathrm{mg} / \mathrm{dl})$ & - & 5.6 \\
\hline PTH (Ref: 9-77 pg/mL) & 69 & 78 \\
\hline TSH (Ref: $0.400-4.500 \mathrm{ulU} / \mathrm{mL})$ & 3.630 & - \\
\hline
\end{tabular}

The patient was immediately transferred to the Department of Endocrine Surgery where the right-inferior parathyroid gland and part of the surrounding lobe of the thyroid were removed without incident. Postoperatively patient exhibited improvement in her laboratory values (Table 2) and was brought back to the geriatric unit where she was noted the following morning to be ambulating on her own around the unit chatting pleasantly with nursing staff and other patients, smiling and laughing appropriately. The patient's presentation was dramatically changed. Intravenous fluids were discontinued when patient's by mouth intake was sufficiently normalized. Unfortunately the dramatic reversal of symptoms was short lived; the patient once again developed a very anxious and negative outlook and it became clear the patient continued to deal with a significant adjustment disorder due to multiple psychosocial stressors she was attempting to cope with. The patient's antidepressant was augmented with Olanzapine $2.5 \mathrm{mg}$ by mouth at bedtime and her sleep and appetite improved further. The patient's severity of symptoms by her statements decreased from a 9-10 out of 10 with 10 being the worst at admission, to a 4 out of 10 after her surgery and later 2 out of 10 prior to discharge.

Table 2. Laboratory examination post-parathyroidectomy and one year post-operative.

\section{Lab Value}

Post-Operative

One Year Post-

Operative

\begin{tabular}{lll}
\hline Calcium (Ref: 8.5-10.4 mg/dl) & 8.9 & 8.7 \\
\hline Calcium lonized (Ref: $4.5-5.3 \mathrm{mg} / \mathrm{dl})$ & 4.8 & - \\
\hline PTH (Ref: $9-77 \mathrm{pg} / \mathrm{mL})$ & 63 & 15 \\
\hline TSH (Ref: $0.400-4.500 \mathrm{ulU} / \mathrm{mL})$ & 4.597 & 3.465 \\
\hline
\end{tabular}

As illustrated by the post-operative laboratory values in this case and also has been emphasized in previous studies, parathyroidectomy has shown to improve overall health, particularly in older people and poses little or no increased risk of complications, with outcomes comparable to those for younger patients $[24,25]$.

\section{Discussion}

As illustrated by this case, PHPT causes significant but often nonspecific psychiatric and somatic symptoms. In this case, initial laboratory values were masked by the patient's profound dehydration and malnutrition, which has been shown to be characteristic of PHPT in older patients. However, this case exemplifies the dynamic nature of working with a medically complex psychiatric patient and how the patient-centered collaborative care approach is an effective model for integrating the skill mix needed to best meet patient and family needs. This model further illustrates that the efficacy of patient-centered collaborative care for co-morbidity goes beyond common psychiatric disorders as establish in a recent meta-analysis of practice-based interventions for depression concomitant with a range of chronic medical conditions [26]. Watson et al. found that collaborative care interventions improved outcomes for depression and quality of life in primary care patients with a number of medical issues. Recognizing that patients with mental illness may also have other medical co-morbidities is essential to maximizing treatment outcomes and enhancing the patient's quality of life. Postoperatively, our patient's recovery was complicated by an underlying adjustment disorder and maladaptive personality traits, although the patient's symptoms became more amenable to therapy and psychopharmacological treatment after normalization of serum calcium and PTH. Hence, patientcentered collaborative care provides an opportunity for collaboration across disciplines to ensure we are coming to the correct diagnoses and subsequent decisions on surgical intervention, treatment and follow-up. As such, when there is no clear answer to the clinical riddle a patient presents to us, it is crucial to unceasingly question the available data. As clinicians we must frequently revisit and refine the differential diagnosis as new information becomes available. In this manner we might hope to avoid inelegant and suboptimal treatments, decrease the number of inpatient hospital days and improve patient outcomes.

\section{Lessons Learned}

At the conclusion of Mrs. Brown's hospitalization there were several observations that stood out as opportunities for improvement/optimization for future patient care. Firstly, when dealing with complicated patients such as in our case there is often an emphasis to arrive quickly at a diagnosis and begin 
Citation: Saul JR, Holder SM, Lokey JS. Employing the patient-centered collaborative care approach: a case study of complex geriatric patient with psychopathalogy of treatment resistant depression and primary hyperparathyroidism. J Ment Health Aging 2018;2(1): $18-23$.

treatment; after all, we do not want to prolong suffering. However, if we rush to what looks like an obvious diagnosis we may miss co-occurring issues or underlying abnormalities. In the words of a respected clinical mentor "Don't just do something, stand there!" Thus, it is crucial to revisit the working differential as treatment progresses; maintaining a dynamic approach to clinical judgment and diagnosis. Secondly, no standardized objective index was used to assess level of severity of the patient's presenting symptoms. Utilizing a tool such as the Geriatric Depression Scale (GDS), Patient Health Questionnaire (PHQ-9), Hamilton Depression Rating Scale (HAM-D) or the health-related quality of life (HRQL) at admission, and again prior to discharge is a fast and powerful way to track patient response to treatment. This also adds further evidence based validity to practice patterns and to later research efforts. Depression occurs in nearly one-third of PHPT patients, with up to $10 \%$ of patients meeting criteria for Major Depression; utilizing PHQ-9 to track depressive symptoms one study found there to be a significant and sustained improvement in depressive symptoms as measured by PHQ-9 scores after parathyroidectomy compared to other treatment options alone [27]. We should therefore, not be hesitant to pursue surgical options if the clinical findings support it. Finally, in the setting of psychiatric care for the older adult, hypercalcemia should be thoroughly investigated by obtaining a corrected serum calcium level then a PTH level which if high-normal or elevated, is indicative of possible PHPT; while if low-normal to low suggests non-PTH cause of hypercalcemia such as malignancy [28].

\section{Conclusion}

In summary, while our patient was medically complex, yet manageable, it is crucial that we be mindful of our limitations as clinicians and knowledgeable of our available resources and how to best utilize them. Collaborating with the available team members, such as our medical and surgical consultants for secondary assessment resulted in early recognition of the parathyroid adenoma. Finding the tumor quickly likely resulted in fewer failed treatments, less risk to patient, reduced costs to consumer and health system, and fewer inpatient hospital days. Mrs. Brown and her family members endorsed being pleased with her treatment team and the care they provided, we recognize that we must continually seek to identify ways to improve our care and outcomes; we are confident that utilizing the patient-centered collaborative care model is one way to do so.

\section{References}

1. Adami S, Marcocci C, Gatti D. Epidemiology of primary hyperparathyroidism in Europe. $\mathrm{J}$ Bone Miner Res. 2002;N18-23.

2. Conroy S, Moulias S, Wassif WS. Primary hyperparathyroidism in the older person. Age Ageing. 2002;32:571-8.

3. Dobrinja C, Silvestri M, Manzini N. Primary hyperparathyroidism in older people: surgical treatment with minimally invasive approaches and outcome. Int $\mathrm{J}$ Endocrinol. 2012.

4. Coker L, Rorie H, Cantley K, et al. Primary hyperparathyroidism, cognition and health-related quality of life. Ann Surg. 2005;242(5):642-50.

5. Grønli O, Wynn R. Normocalcemic hyperparathyroidism and treatment resistive depression. Psychosomatics. 2013;54(4):493-7.

6. Morris LF, Zelada J, Wu B, et al. Parathyroid surgery in the elderly. Oncologist. 2010;15(12):1273-84.

7. Sheldon DG, Lee FT, Neil NJ, et al. Surgical treatment of hyperparathyroidism improves health-related quality of life. Arch Surg. 2002;137:1022-6.

8. Pasieka JL, Parsons L, Jones J. The long-term benefit of parathyroidectomy in primary hyperparathyroidism: A 10 year prospective surgical outcome study. Surgery. 2009; 146:1006-13.

9. Rubin MR, Bilezikian JP, McMahon DJ, et al. The natural history of primary hyperparathyroidism with or without parathyroid surgery after 15 years. J Clin Endocrinol Metab. 2008;93:3462-70.

10. Chiba Y, Satoh K, Ueda S, et al. Marked improvement of psychiatric symptoms after parathyroidectomy in elderly primary hyperparathyroidism. Endocr J. 2007;54(3): 379-83.

11. Sevin C, Moore G, Shepherd J, et al. Transforming care teams to provide the best possible patient-centered, collaborative care. J Ambul Care Manage. 2009;32(1): 24-31.

12. Institute of Medicine. Crossing the quality chasm: A new health system for the 21 st century. Washington DC: National Academy Press. 2009.

13. Katon WJ, Lin EHB, Von Kroff M, et al. Collaborative care for patients with depression and chronic illnesses. $\mathrm{N}$ Engl J Med. 2010;363:2611-20.

14. Epstein RM, Street RL. The values and value of patientcentered care. Ann Fam Med. 2011;9(2):100-3.

15. Berwick DM. What "patient-centered" should mean: confessions of an extremist. Health Affairs. 2009:28(4): 555-65.

16. Gilbody S, Bower P, Fletcher J, et al. Collaborative care for depression: a cumulative meta-analysis and review of longer-term outcomes. Arch Intern Med. 2006; 166:2314-21.

17. Stewart M, Brown JB, Donner A. The impact of patientcentered care on outcomes. J Fam Pract. 2000;49:796-804.

18. Schottenfeld L, Petersen D, Peikes D, et al. Creating patient-centered team-based primary care. AHRQ Pub. No. 16-0002-EF. Rockville, MD: Agency for Healthcare Research and Quality. March 2016.

19. Bechtel C, Ness DL. If you build it, will they come? Designing truly patient-centered health care. Health Affairs. 2010;29(5):914-20.

20. Marmot M. Fair society, healthy lives: The marmot review. 2010. 
21. Unützer J, Park M. Older adults with severe, treatmentresistant depression: "I got my mother back". JAMA. 2012;308(9):909-18.

22. Dombrovski AY, Mulsant BH. ECT: the preferred treatment for severe depression in late life. Int Psychogeriatr. 2007;19(1):10-4.

23. Politz D, Norman J. Hyperparathyroidism in patients over 80: Clinical characteristics and their ability to undergo outpatient parathyroidectomy. Thyroid. 2007;17:333-9.

24. Kebebew E, Duh QY, Clark, OH. Parathyroidectomy for primary hyperparathyroidism in octogenarians and nonagenarians. Arch Surg. 2003;138:867-71.

25. Watson LC, Amick HR, Gaynes BN, et al. Practice-based interventions addressing concomitant depression and chronic medical conditions in the primary care setting: a systematic review and meta-analysis. J Prim Care Community Health. 2013;4(4):294-306.

26. Watson LC, Amick HR, Gaynes BN, et al. Practice-based interventions addressing concomitant depression and chronic medical conditions in the primary care setting: A systematic review and meta-analysis. J Prim Care Community Health. 2013; 4(4):294-306.

27. Espiritu RP, Kearns AE, Vickers KS, et al. Depression in primary hyperparathyroidism: Prevalence and benefit of surgery. J Clin Endocrinol Metab. 2011;96(11):E1737-45.

28. Lafferty FW. Differential diagnosis of hypercalcemia. J Bone Miner Res. 1991;6:S51.

\section{*Correspondence to:}

Sharon M Holder

Clemson University

South Carolina, USA

E-mail: sholder2@ghs.org 